\title{
The Effect of Spatial Averaging on Spectrum Estimation
}

\author{
BERNARD A. STLVERMAN ${ }^{1}$ \\ Air Force Cambridge Research Laboratories, Bedjord, Mass. \\ (Manuscript received 6 October 1967)
}

\begin{abstract}
The effect of instrument baseline averaging on the estimated spectrum density of a homogeneous random field of a scalar variable in turbulent flow is investigated. The spectrum transfer function of the spatial filter is evaluated for all orientations of the baseline to the vector mean wind. It is shown that the influence of baseline averaging on the spectrum increases as the angle between the baseline and the vector mean wind decreases and as the ratio of the baseline length to the wavelength of the fluctuations increases. These results can be used to correct the computed spectra of spatially averaged components of the velocity field in turbulent flow and of spatially averaged conservative passive additives in a turbulent flow.
\end{abstract}

\section{Introduction}

There are some variables in atmospheric research which are measured over an instrument baseline, e.g., sonic anemometer measurements of the wind velocity components, infrared hygrometer measurements of the absolute humidity, and optical transmissometer measurements of the extinction coefficient. Fluctuations corresponding to eddy diameters smaller than the baseline are, therefore, attenuated. To obtain an unbiased estimate of the spectrum density of variables measured in this manner, it is necessary to correct the computed spectrum for the effect of the baseline averaging. Since the averaging is purely spatial, its influence on the spatial spectrum of the variable can be determined and interpreted in terms of the frequency spectrum by applying Taylor's hypothesis of frozen turbulence (Taylor, 1938). The magnitude of the spectrum attenuation due to spatial averaging as a function of wavenumber or frequency depends on the length of the baseline and its orientation to the vector mean wind. Gurvich (1962) derived and evaluated the spectrum transfer function due to baseline averaging in sonic anemometer measurements of vertical velocity, i.e., where the baseline is normal to the vector mean wind. Mitsuta (1966) calculated the frequency response function due to baseline averaging in sonic anemometer-thermometer measurements where the baseline is coincident with the vector mean wind.

It is the purpose of this paper to obtain a general solution for the effect of spatial averaging on the spectrum of a homogeneous random field of a scalar variable in turbulent flow. The spectrum transfer function is evaluated for all orientations of the baseline to the vector mean wind. The particular cases considered by

\footnotetext{
${ }^{1}$ Much of the work reported in this paper was accomplished while the author was attending The University of Chicago under Air Force sponsorship.
}

Gurvich and Mitsuta are included in the general solution.

\section{Spectrum of homogeneous random scalar field}

Consider the scalar quantity $X$ which is a random function of three space variables, i.e., the random field $X(r)$. In the case of random fields the concept of stationarity in time, a basic assumption in spectrum analysis, generalizes to the concept of homogeneity. A random field is called homogeneous if its mean value is constant and if its correlation function depends only on the separation between points in a given direction. Without loss of generality, only zero-mean, homogeneous random fields will be considered here.

A homogeneous random field can be represented in the form of a three-dimensional stochastic FourierStieltjes integral,

$$
X(\mathbf{r})=\iint_{-\infty}^{\infty} \int e^{i \mathbf{k} \cdot \mathbf{r}} d Z(\mathbf{k}),
$$

where $\mathbf{k}$ is a radian wavenumber vector $\left(k_{1}, k_{2}\right.$ and $k_{3}$ are the radian wavenumber components along the coordinate axes $x, y$ and $z$, respectively). $Z(\mathrm{k})$ is a complex, random function with orthogonal increments

$$
\begin{aligned}
\overline{d Z(\mathbf{k}) d Z^{\cdot}\left(\mathbf{k}^{\prime}\right)}=\left\{\begin{array}{cc}
0, & \mathbf{k} \neq \mathbf{k}^{\prime} \\
d \Phi(\mathbf{k}), & \mathbf{k}=\mathbf{k}^{\prime}
\end{array}\right\}, \\
d \Phi(\mathbf{k})=F_{\mathrm{a}}(\mathbf{k}) d \mathbf{k},
\end{aligned}
$$

where the asterisk denotes complex conjugate and the overbar denotes the averaging process. $\Phi(\mathbf{k})$ and $F_{3}(\mathbf{k})$ are the three-dimensional spectrum distribution function and the three-dimensional spectrum density function of the homogeneous random scalar field $X(\mathbf{r})$, 
respectively. Since $Z(\mathbf{k})=\frac{1}{2}[U(\mathbf{k})+i V(\mathbf{k})]$, where $U(\mathbf{k})$ is an even function and $V(\mathbf{k})$ is an odd function, for real processes (1) becomes

$$
X(\mathbf{r})=\iint_{0}^{\infty} \int[\cos (\mathbf{k} \cdot \mathbf{r}) d U(\mathbf{k})
$$

$$
+\sin (\mathbf{k} \cdot \mathbf{r}) d V(\mathbf{k})] \text {. }
$$

By taking into account the relation (2), the correlation function $C(\mathbf{r})$ can be expressed as a function of $F_{3}(\mathbf{k})$, i.e.,

$$
C(\mathbf{r})=\iint_{-\infty}^{\infty} \int^{i \mathbf{k} \cdot \mathbf{r}} F_{3}(\mathbf{k}) d \mathbf{k} .
$$

Since $C(\mathbf{r})$ and $F_{3}(\mathbf{k})$ are Fourier transforms of each other, we have

$$
F_{3}(\mathbf{k})=\frac{1}{(2 \pi)^{3}} \iint_{-\infty}^{\infty} \int e^{-i \mathbf{k} \cdot \mathbf{r}} C(\mathbf{r}) d \mathbf{r}
$$

\section{Spectrum of a spatially averaged variable}

For mathematical convenience, let the $x$ axis be directed along the vector mean wind. In addition, since the effect of spatial averaging depends only on the angle between the baseline and the vector mean wind regardless of its absolute orientation, we shall only consider the case where the baseline is in the $x, y$ plane for computational purposes.

The instantaneous value of the measurement is proportional to the average value of the variable over the instrument baseline. The spatially averaged measurements can therefore be represented by

$$
\widetilde{X}(\mathbf{r})=\frac{1}{s} \int_{s} X(\mathbf{r}) d s,
$$

where $s$ is the length of the baseline. Substituting (3) into (6) and evaluating it along the $x$ axis at the center point of the baseline, we obtain

$$
\begin{aligned}
& \widetilde{X}(\mathbf{r})=\frac{1}{s \cos \theta} \\
& \quad \times \int_{x-(s / 2) \cos \theta}^{x+(s / 2) \cos \theta} \iint_{0}^{\infty} \int\left\{\cos \left[x\left(k_{1}+k_{2} \tan \theta\right)+k_{3} z\right] d U(\mathbf{k})\right. \\
& \left.\quad+\sin \left[x\left(k_{1}+k_{2} \tan \theta\right)+k_{3} z\right] d V(\mathbf{k})\right\} d x, \quad \text { (7) }
\end{aligned}
$$

where $\theta$ is the angle between the baseline and the vector mean wind. Integrating (7) over $x$ and collecting terms we obtain

$$
\tilde{X}(\mathbf{r})=A(\mathbf{k}, \theta) X(\mathbf{r}),
$$

where $A(\mathbf{k}, \theta)$ is called the wavenumber response func- tion of the spatial filter corresponding to the baseline averaging and is given by

$$
A(\mathbf{k}, \theta)=\frac{\sin \left[k_{1}(s / 2) \cos \theta+k_{2}(s / 2) \sin \theta\right]}{\left[k_{1}(s / 2) \cos \theta+k_{2}(s / 2) \sin \theta\right]} .
$$

When the baseline is directed along the vector mean wind, i.e., $\theta=0^{\circ}$, (9) reduces to the wavenumber response function corresponding to the frequency response function obtained by Mitsuta, i.e.,

$$
A\left(\mathbf{k}, 0^{\circ}\right)=\frac{\sin \left[k_{1}(s / 2)\right]}{\left[k_{1}(s / 2)\right]} .
$$

Using the relation (8), we obtain the correlation function of the measured variable along the $x$ axis,

$$
\widetilde{C}(x, 0,0)=\iint_{-\infty}^{\infty} \int A^{2}(\mathbf{k}, \theta) e^{i k 1 x} F_{3}(\mathbf{k}) d \mathbf{k} .
$$

Thus, the three-dimensional spectrum density of $\widetilde{C}$ differs from the true spectrum density by the factor $A^{2}(\mathbf{k}, \theta)$, the transfer function of the spatial filter.

Since we can only obtain an estimate of the one-dimensional spatial spectrum density along the $x$ axis from the measurements, we obtain the relation

$$
\tilde{F}_{1}(\xi)=\frac{1}{2 \pi} \int_{-\infty}^{\infty} \tilde{C}(x, 0,0) e^{-i \xi x} d x,
$$

where $\widetilde{F}_{1}(\xi)$ is the one-dimensional spatial spectrum density that corresponds, through Taylor's hypothesis, to the one-dimensional frequency spectrum density of the observed time series, i.e., $\widetilde{F}_{1}(\xi)=\bar{V} F_{1}(\omega), \xi=\omega / \bar{V}$, where $\omega$ is the angular frequency and $\bar{V}$ is the magnitude of the vector mean wind. Substituting (11) into (12) yields

$$
\begin{aligned}
\tilde{F}_{1}(\xi)=\frac{1}{2 \pi} \iiint_{-\infty}^{\infty} \int & A^{2}(\mathbf{k}, \theta) \\
& \times e^{i\left(k_{1}-\xi\right) x} F_{3}(\mathbf{k}) d k_{1} d k_{2} d k_{3} d x .
\end{aligned}
$$

After making the variable transformation, $k_{1}^{\prime}=k_{1}-\xi$, and integrating (13) over $x$ and $k_{1}{ }^{\prime}$ we obtain

$$
\tilde{F}_{1}(\xi)=\int_{-\infty}^{\infty} \int^{\infty} F_{3}\left(\xi, k_{2}, k_{3}\right) B^{2}\left(\xi, k_{2}, \theta\right) d k_{2} d k_{3}
$$

where

$$
B\left(\xi, k_{2}, \theta\right)=\frac{\sin \left[\xi(s / 2) \cos \theta+k_{2}(s / 2) \sin \theta\right]}{\left[\xi(s / 2) \cos \theta+k_{2}(s / 2) \sin \theta\right]} .
$$

To determine the spectrum transfer function due to spatial averaging, it is necessary to integrate (14) over $k_{2}$ and $k_{3}$ for the various values of $\theta$ and compare it to 
TABde 1. Values of the spectrum transfer function $T(s / \lambda, \theta)$.

\begin{tabular}{|c|c|c|c|c|c|c|c|c|c|c|c|c|}
\hline$(s / \lambda)$ & $0^{\circ}$ & $10^{\circ}$ & $15^{\circ}$ & $20^{\circ}$ & $25^{\circ}$ & $\begin{array}{r}\theta \\
30^{\circ} \\
\end{array}$ & $40^{\circ}$ & $50^{\circ}$ & $60^{\circ}$ & $70^{\circ}$ & $80^{\circ}$ & $90^{\circ}$ \\
\hline 0. & 1.0000 & 1.0000 & 1.0000 & 1.0000 & 1.0000 & 1.0000 & 1.0000 & 1.0000 & 1.0000 & 1.0000 & 1.0000 & 1.0000 \\
\hline 0.064 & 0.9867 & 0.9845 & 0.9827 & 0.9794 & 0.9770 & 0.9745 & 0.9695 & 0.9656 & 0.9617 & 0.9592 & 0.9576 & 0.9562 \\
\hline 0.127 & 0.9478 & 0.9412 & 0.9362 & 0.9309 & 0.9263 & 0.9212 & 0.9124 & 0.9052 & 0.8998 & 0.8959 & 0.8938 & 0.8929 \\
\hline 0.191 & 0.8856 & 0.8759 & 0.8700 & 0.8634 & 0.8577 & 0.8521 & 0.8432 & 0.8365 & 0.8324 & 0.8297 & 0.8279 & 0.8275 \\
\hline 0.255 & 0.8041 & 0.7937 & 0.7883 & 0.7823 & 0.7782 & 0.7745 & 0.7695 & 0.7674 & 0.7658 & 0.7652 & 0.7656 & 0.7656 \\
\hline 0.318 & 0.7081 & 0.7011 & 0.6975 & 0.6958 & 0.6949 & 0.6943 & 0.6961 & 0.6996 & 0.7032 & 0.7055 & 0.7072 & 0.7083 \\
\hline 0.382 & 0.6033 & 0.6021 & 0.6038 & 0.6070 & 0.6110 & 0.6159 & 0.6257 & 0.6359 & 0.6445 & 0.6515 & 0.6550 & 0.6553 \\
\hline 0.446 & 0.4955 & 0.5035 & 0.5116 & 0.5208 & 0.5309 & 0.5414 & 0.5607 & 0.5779 & 0.5916 & 0.6019 & 0.6085 & 0.6102 \\
\hline 0.509 & 0.3903 & 0.4099 & 0.4249 & 0.4415 & 0.4578 & 0.4735 & 0.5021 & 0.5260 & 0.5445 & 0.5580 & 0.5653 & 0.5686 \\
\hline 0.573 & 0.2927 & 0.3250 & 0.3473 & 0.3702 & 0.3922 & 0.4130 & 0.4501 & 0.4799 & 0.5027 & 0.5188 & 0.5274 & 0.5314 \\
\hline 0.637 & 0.2067 & 0.2515 & 0.2803 & 0.3094 & 0.3363 & 0.3612 & 0.4046 & 0.4391 & 0.4652 & 0.4835 & 0.4944 & 0.4981 \\
\hline 0.700 & 0.1351 & 0.9101 & 0.2247 & 0.2577 & 0.2885 & 0.3167 & 0.3650 & 0.4032 & 0.4321 & 0.4526 & 0.4041 & 0.4681 \\
\hline 0.764 & 0.0792 & 0.1416 & 0.1796 & 0.2156 & 0.2489 & 0.2793 & 0.3311 & 0.3719 & 0.4027 & 0.4243 & 0.4369 & 0.4412 \\
\hline 0.828 & 0.0393 & 0.1053 & 0.1447 & 0.1821 & 0.2166 & 0.2480 & 0.3020 & 0.3444 & 0.3766 & 0.3993 & 0.4124 & 0.4169 \\
\hline 0.891 & 0.0143 & 0.0798 & 0.1189 & 0.1561 & 0.1908 & 0.2224 & 0.2772 & 0.3203 & 0.3535 & 0.3767 & 0.3905 & 0.3952 \\
\hline 0.955 & 0.0022 & 0.0633 & 0.1005 & 0.1364 & 0.1701 & 0.2012 & 0.2556 & 0.2993 & 0.3327 & 0.3564 & 0.3707 & 0.3753 \\
\hline 1.000 & 0.0000 & 0.0561 & 0.0911 & 0.1254 & 0.1581 & 0.1885 & 0.2422 & 0.2859 & 0.3195 & 0.3433 & 0.3575 & 0.3623 \\
\hline 1.019 & 0.0003 & 0.0540 & 0.0880 & 0.1215 & 0.1536 & 0.1837 & 0.2371 & 0.2807 & 0.3143 & 0.3382 & 0.3524 & 0.3572 \\
\hline 1.082 & 0.0056 & 0.0498 & 0.0798 & 0.1104 & 0.1405 & 0.1692 & 0.2210 & 0.2639 & 0.2973 & 0.3215 & 0.3358 & 0.3406 \\
\hline 1.146 & 0.0151 & 0.0487 & 0.0745 & 0.1020 & 0.1299 & 0.1571 & 0.2070 & 0.2492 & 0.2824 & 0.3064 & 0.3206 & 0.3254 \\
\hline 1.210 & 0.0259 & 0.0492 & 0.0709 & 0.0955 & 0.1211 & 0.1468 & 0.1948 & 0.2360 & 0.2687 & 0.2925 & 0.3067 & 0.3114 \\
\hline 1.273 & 0.0358 & 0.0501 & 0.0683 & 0.0903 & 0.1140 & 0.1381 & 0.1842 & 0.2241 & 0.2563 & 0.2796 & 0.2939 & 0.2986 \\
\hline 1.337 & 0.0431 & 0.0468 & 0.0660 & 0.0857 & 0.1077 & 0.1304 & 0.1746 & 0.2134 & 0.2451 & 0.2679 & 0.2821 & 0.2867 \\
\hline 1.401 & 0.0468 & 0.0503 & 0.0636 & 0.0816 & 0.1021 & 0.1236 & 0.1660 & 0.2037 & 0.2347 & 0.2572 & 0.2712 & 0.2757 \\
\hline 1.464 & 0.0467 & 0.0488 & 0.0609 & 0.0777 & 0.0970 & 0.1175 & 0.1852 & 0.1950 & 0.2252 & 0.2472 & 0.2611 & 0.2654 \\
\hline 1.528 & 0.0431 & 0.0464 & 0.0580 & 0.0739 & 0.0923 & 0.1119 & 0.1512 & 0.1869 & 0.2164 & 0.2380 & 0.2515 & 0.2560 \\
\hline 1.592 & 0.0368 & 0.0432 & 0.0549 & 0.0703 & 0.0879 & 0.1068 & 0.1448 & 0.1794 & 0.2083 & 0.2296 & 0.2427 & 0.2471 \\
\hline 1.751 & 0.0165 & 0.0339 & 0.0469 & 0.0619 & 0.0783 & 0.0956 & 0.1307 & 0.1631 & 0.1903 & 0.2106 & 0.2232 & 0.2274 \\
\hline 1.910 & 0.0022 & 0.0265 & 0.0402 & 0.0548 & 0.0703 & 0.0865 & 0.1192 & 0.1495 & 0.1752 & 0.1945 & 0.2065 & 0.2105 \\
\hline 2.000 & 0.0000 & 0.0238 & 0.0372 & 0.0514 & 0.0664 & 0.0820 & 0.1135 & 0.1429 & 0.1677 & 0.1865 & 0.1981 & 0.2020 \\
\hline 2.069 & 0.0011 & 0.0225 & 0.0353 & 0.0491 & 0.0637 & 0.0789 & 0.1095 & 0.1381 & 0.1624 & 0.1807 & 0.1921 & 0.1960 \\
\hline 2.228 & 0.0088 & 0.0211 & 0.0321 & 0.0447 & 0.0583 & 0.0725 & 0.1013 & 0.1283 & 0.1513 & 0.1687 & 0.1796 & 0.1833 \\
\hline 2.387 & 0.0156 & 0.0203 & 0.0296 & 0.0411 & 0.0537 & 0.0670 & 0.0941 & 0.1197 & 0.1416 & 0.1582 & 0.1686 & 0.1721 \\
\hline 2.546 & 0.0153 & 0.0190 & 0.0274 & 0.0381 & 0.0499 & 0.0624 & 0.0880 & 0.1123 & 0.1331 & 0.1489 & 0.1588 & 0.1622 \\
\hline 2.706 & 0.0088 & 0.0171 & 0.0253 & 0.0354 & 0.0465 & 0.0584 & 0.0826 & 0.1056 & 0.1255 & 0.1407 & 0.1501 & 0.1534 \\
\hline 2.865 & 0.0021 & 0.0152 & 0.0234 & 0.0330 & 0.0436 & 0.0547 & 0.0778 & 0.0997 & 0.1188 & 0.1333 & 0.1424 & 0.1455 \\
\hline 3.000 & 0.0000 & 0.0139 & 0.0220 & 0.0311 & 0.0413 & 0.0520 & 0.0742 & 0.0952 & 0.1136 & 0.1276 & 0.1363 & 0.1393 \\
\hline 3.024 & 0.0001 & 0.0138 & 0.0217 & 0.0308 & 0.0409 & 0.0516 & 0.0736 & 0.0945 & 0.1126 & 0.1267 & 0.1353 & 0.1383 \\
\hline 3.183 & 0.0030 & 0.0128 & 0.0203 & 0.0290 & 0.0386 & 0.0487 & 0.0698 & 0.0898 & 0.1072 & 0.1207 & 0.1290 & 0.1318 \\
\hline 3.324 & 0.0070 & 0.0122 & 0.0191 & 0.0274 & 0.0365 & 0.0462 & 0.0663 & 0.0856 & 0.1022 & 0.1152 & 0.1233 & 0.1259 \\
\hline 3.501 & 0.0083 & 0.0116 & 0.0181 & 0.0259 & 0.0346 & 0.0439 & 0.0632 & 0.0817 & 0.0977 & 0.1102 & 0.1180 & 0.1205 \\
\hline 3.661 & 0.0058 & 0.0109 & 0.0171 & 0.0246 & 0.0330 & 0.0419 & 0.0603 & 0.0781 & 0.0935 & 0.1056 & 0.1131 & 0.1156 \\
\hline 3.820 & 0.0020 & 0.0102 & 0.0162 & 0.0234 & 0.0314 & 0.0400 & 0.0577 & 0.0749 & 0.0897 & 0.1012 & 0.1086 & 0.1110 \\
\hline 3.979 & 0.0000 & 0.0095 & 0.0154 & 0.0223 & 0.0300 & 0.0383 & 0.0554 & 0.0719 & 0.0862 & 0.0973 & 0.1045 & 0.1068 \\
\hline 4.000 & 0.0000 & 0.0095 & 0.0153 & 0.0222 & 0.0299 & 0.0381 & 0.0551 & 0.0715 & 0.0858 & 0.0968 & 0.1040 & 0.1063 \\
\hline 4.138 & 0.0010 & 0.0090 & 0.0147 & 0.0213 & 0.0287 & 0.0367 & 0.0532 & 0.0691 & 0.0830 & 0.0937 & 0.1007 & 0.1030 \\
\hline 4.297 & 0.0035 & 0.0086 & 0.0140 & 0.0204 & 0.0276 & 0.0352 & 0.0511 & 0.0665 & 0.0800 & 0.0904 & 0.0971 & 0.0993 \\
\hline 4.456 & 0.0050 & 0.0083 & 0.0134 & 0.0196 & 0.0265 & 0.0339 & 0.0493 & 0.0641 & 0.0772 & 0.0873 & 0.0938 & 0.0959 \\
\hline 4.615 & 0.0042 & 0.0079 & 0.0129 & 0.0188 & 0.0255 & 0.0326 & 0.0475 & 0.0619 & 0.0746 & 0.0843 & 0.0907 & 0.0928 \\
\hline 4.775 & 0.0019 & 0.0075 & 0.0123 & 0.0181 & 0.0245 & 0.0315 & 0.0459 & 0.0599 & 0.0722 & 0.0816 & 0.0878 & 0.0898 \\
\hline
\end{tabular}

the one-dimensional spectrum density of the non- therefore given by averaged variable, $F_{1}(\xi)$.

\section{Spectrum transfer function}

$T(\xi s / 2, \theta)=\frac{\tilde{F}_{1}(\xi)}{F_{1}(\xi)}=\left[\frac{\sin (\xi s / 2)}{(\xi s / 2)}\right]^{2}, \quad \theta=0^{\circ}$,

Consider first the particular case where $\theta=0^{\circ}$, i.e. where the baseline is along the $x$ axis. Then (14) reduces to

$$
\begin{aligned}
\widetilde{F}_{\mathbf{1}}(\xi)=\left[\frac{\sin (\xi s / 2)}{(\xi s / 2)}\right]^{2} \int_{-\infty}^{\infty} \int_{3} F_{3}\left(\xi, k_{2}, k_{3}\right) d k_{2} d k_{3} \\
=\left[\frac{\sin (\xi s / 2)}{(\xi s / 2)}\right]^{2} F_{\mathbf{1}}(\xi) .
\end{aligned}
$$

The spectrum transfer function, $T(\xi s / 2, \theta)$ at $\theta=0^{\circ}$ is

and can be evaluated explicitly.

For the case where $0^{\circ}<\theta \leq 90^{\circ}$, it is necessary to simplify (14) by substituting an appropriate form for $F_{3}\left(\xi, k_{2} k_{3}\right)$ in order to facilitate the integration. On the assumption that the homogeneous random field of the scalar variable follows the Kolmogorov law, we can use

$$
F_{3}(\mathbf{k})=C^{2} k^{-11 / 3} \text {. }
$$

Tatarskii (1961) has shown that this form of $F_{3}(\mathbf{k})$ is equally appropriate for the components of the velocity field in turbulent flow and the field of a conservative 


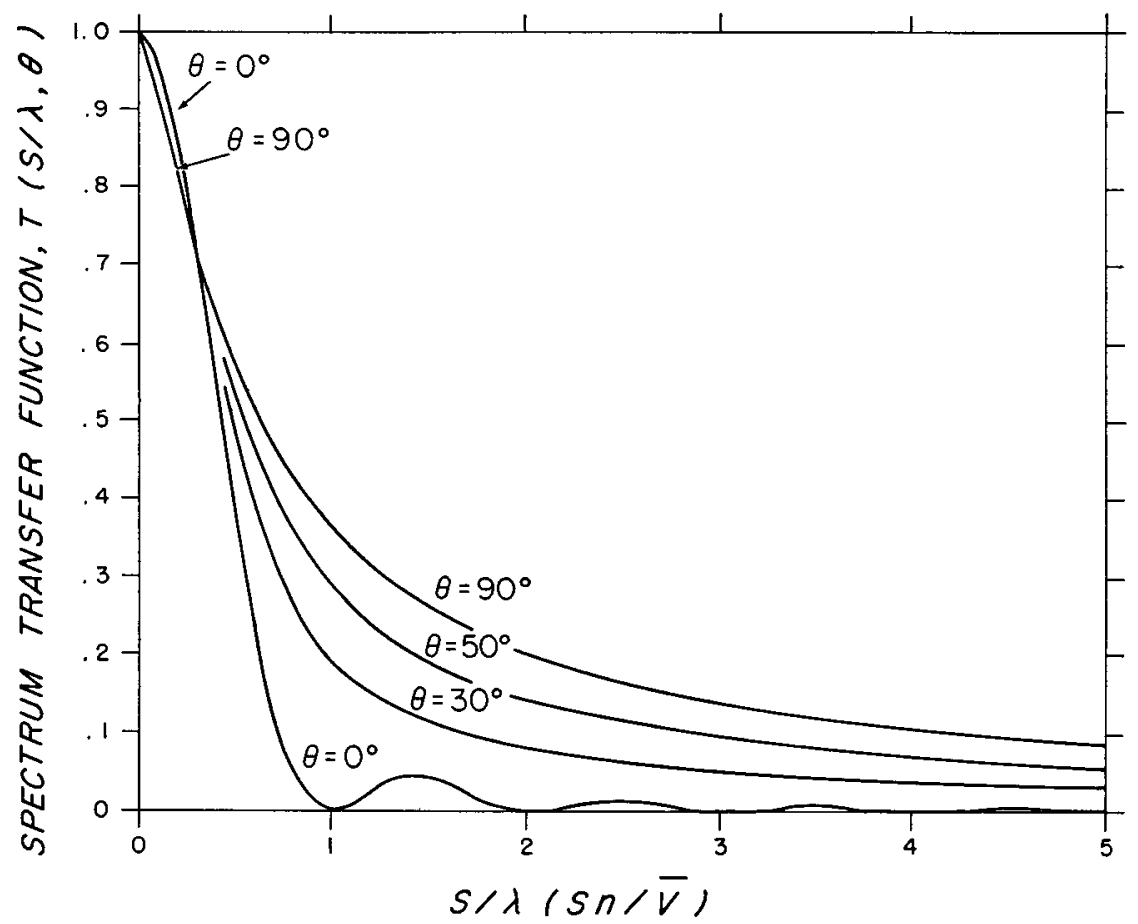

FIG. 1. Plot of the spectrum transfer function vs the nondimensional variable $(s / \lambda)$ for selected values of the angle between the baseline and the vector mean wind $\left(\theta=0^{\circ}, 30^{\circ}, 50^{\circ}\right.$ and $90^{\circ}$ ) where $s$ is the length of the instrument baseline, $\lambda$ the wavelength, $n$ the cyclic frequency, and $\bar{V}$ the magnitude of the vector mean wind. The spectrum transfer function represents the fraction of the true one-dimensional spectrum that is transmitted by the spatial filter.

passive additive in a turbulent flow. An additive in a volume of air is considered conservative if it does not change when the volume element is shifted about in space. It is considered passive if its presence in the air does not affect the dynamical regime of the turbulence.

Substituting (17) into (14) we obtain

$$
\tilde{F}_{\mathbf{1}}(\xi)=C^{2} \int_{-\infty}^{\infty} \int_{-\infty}\left(\xi^{2}+k_{2}{ }^{2}+k_{3}{ }^{2}\right)^{-11 / 6} E^{2}\left(\xi, k_{2}, \theta\right) d k_{2} d k_{3},
$$

which, to facilitate integration, is transformed into

$$
\begin{aligned}
\tilde{F}_{1}(\xi)=C^{2} \xi^{-5 / 3} & \int_{-\infty}^{\infty}\left(1+\frac{k_{2}^{2}}{\xi^{2}}\right)^{-4 / 3} B^{2}\left(\xi, k_{2}, \theta\right) \frac{d k_{2}}{\xi} \\
& \times \int_{-\infty}^{\infty}\left(1+\frac{k_{3}^{2}}{\xi^{2}+k_{2}^{2}}\right)^{-11 / 6} \frac{d k_{3}}{\left(\xi^{2}+k_{2}^{2}\right)^{\frac{1}{2}}}
\end{aligned}
$$

Using the variable transformation $x=k_{3}{ }^{2} /\left(\xi^{2}+k_{2}{ }^{2}\right)$ to evaluate the second integral in (18) we obtain

$$
\begin{aligned}
\widetilde{F}_{1}(\xi)=\frac{\sqrt{\pi} \Gamma(4 / 3)}{\Gamma(11 / 6)} & C^{2} \xi^{-5 / 3} \\
& \times \int_{-\infty}^{\infty}\left(1+\frac{k_{2}^{2}}{\xi^{2}}\right)^{-4 / 3} B^{2}\left(\xi, k_{2}, \theta\right) \frac{d k_{2}}{\xi} .
\end{aligned}
$$

If there is no baseline averaging, i.e., if $B^{2}\left(\xi, k_{2}, \theta\right)=1$, the integral in (19) can be evaluated by using the vari- able transformation $x=k_{2}{ }^{2} / \xi^{2}$ to give the true one-dimensional spectrum density $F_{1}(\xi)$, i.e.,

$$
F_{1}(\xi)=\frac{6 \pi}{5} C^{2} \xi^{-5 / 3}
$$

which is the result one would have expected from the assumed form of $F_{3}(\mathbf{k})$.

Consequently, the spectrum transfer function for $0^{\circ}<\theta \leq 90^{\circ}$ is equal to the ratio of (19) to (20), i.e.,

$$
\begin{aligned}
& T(\xi s / 2, \theta)=\frac{5 \Gamma(4 / 3)}{6 \sqrt{\pi} \Gamma(11 / 6)} \\
& \quad \times \int_{-\infty}^{\infty}\left(1+\frac{k_{2}^{2}}{\xi^{2}}\right)^{-4 / 3} B^{2}\left(\xi, k_{2}, \theta\right) \frac{d k_{2}}{\xi} .
\end{aligned}
$$

Multiplying numerator and denominator by

$$
[(s / 2) \sin \theta]^{-16 / 3}
$$

and letting $x=\xi(s / 2) \cos \theta+k_{2}(s / 2) \sin \theta,(21)$ reduces to a form more suitable for evaluation:

$$
\begin{aligned}
& T(\xi s / 2, \theta)=\frac{5 \Gamma(4 / 3)}{6 \sqrt{\pi} \Gamma(11 / 6)} \frac{1}{\xi(s / 2) \sin \theta} \\
& \times \int_{-\infty}^{\infty}\left\{1+\left[\frac{x-\xi(s / 2) \cos \theta}{\xi(s / 2) \sin \theta}\right]^{2}\right\}^{-4 / 3} \frac{\sin ^{2} x}{x^{2}} d x .
\end{aligned}
$$

Since (22) cannot be reduced any further analytically, 
it is necessary for the integral in (22) to be evaluated by an approximate numerical method. When $\theta=90^{\circ}$, (22) reduces to the form obtained by Gurvich.

\section{Discussion}

Numerical values of the spectrum transfer functions (16) and (22) are presented in Table 1 and plotted in Fig. 1 for selected values of $\theta$. To be of most benefit to the user, the values of the spectrum transfer function are given as a function of the nondimensional variable $s / \lambda$ (or, equivalently, $s n / \bar{V}$ ) where $\lambda$ is the wavelength of the fluctuations and $n$ is the cyclic frequency. The influence of the spatial averaging on the spectrum is seen to increase as the angle $\theta$ between the baseline and the vector mean wind decreases and as the value of $s / \lambda$ increases.

In view of the inevitable inaccuracies in experimental data, it is deemed unrealistic to correct computed spectra by factors larger than ten, the spectrum correction factor being equal to the reciprocal of the particular value of the spectrum transfer function. Wavelengths having values of the spectrum transfer function which are less than the limiting value of 0.1 should therefore be considered to be completely attenuated by the spatial filter, i.e., values of $s / \lambda>0.75$ and $s / \lambda>4.0$ for the limiting cases of $\theta=0^{\circ}$ and $\theta=90^{\circ}$, respectively. For the case of $\theta=0^{\circ}$, the wavelengths corresponding to integer values of $s / \lambda$ are, in fact, completely attenuated since the value of the spectrum transfer function at these values of $s / \lambda$ is zero. It is therefore impossible to obtain corrected estimates of the spectrum density at these wavelengths.

As mentioned before, Gurvich (1962) and Mitsuta (1966) obtained particular solutions of the spectrum transfer function which they used to correct their velocity spectra for the attenuating effect of the sonic- anemometer baseline averaging. Kaimal (1968) has recently obtained experimental evidence to show that the theoretically derived values of the spectrum transfer function can also be used to correct the spectra of spatially averaged temperature measurements. $\mathrm{He}$ found excellent agreement between theory and experiment when he compared the computed spectra of simultaneous measurements of line-averaged temperatures and point temperatures. Thus, it appears that the values of the spectrum transfer function derived here can be used to correct the computed spectra of spatially averaged components of the velocity field in turbulent flow and the computed spectra of spatially averaged, conservative passive additives in a turbulent flow.

Acknowledgments. The author wishes to thank Prof. Roscoe R. Braham, Jr., of the Department of Geophysical Sciences, The University of Chicago, for permitting him to carry out the computations in this paper on the University's IBM 7090-7094 computer. He also wishes to thank Dr. J. Chandran Kaimal, AFCRL, for his invaluable advice in the preparation of this manuscript.

\section{REFERENCES}

Gurvich, A. S., 1962: The pulsation spectra of the vertical component of wind velocity and their relations to the micrometeorological conditions. Akad. Nauk SSSR, Inst. Fiz. Atmos. Tr., No. 4, 101-136 (English translation available from the American Meteorological Society).

Kaimal, J. C., 1968: The effect of vertical line averaging on the spectra of temperature and heat flux. Quart. J. Roy. Meteor. Soc., 94 (accepted for publication).

Mitsuta, Y., 1966: Sonic anemometer-thermometer for general use. J. Meteor. Soc. Japan, Series II, 44, No. 1, 12-23.

Tatarskii, V. I., 1961: Wave Propagation in a Turbulent Medium. Translated by R. A. Silverman, New York, McGraw-Hill Book Co., Inc., 285 pp.

Taylor, G. I., 1938: The spectrum of turbulence. Proc. Roy. Soc. (London), A164, 476-490. 